\title{
A consensus model of political decision-making
}

\author{
Patrik Eklund • Agnieszka Rusinowska • \\ Harrie de Swart
}

Published online: 13 September 2007

(C) Springer Science+Business Media, LLC 2007

\begin{abstract}
In this paper, a model of political consensus is introduced. Parties try to reach consensus in forming a government. A government is defined as a pair consisting of a winning coalition and a policy supported by this coalition, where a policy consists of policies on given issues. A party evaluates all governments the party belongs to with respect to some criteria. We allow the criteria to be of unequal importance to a party. These criteria concern winning coalitions and policy issues. Parties may be advised to adjust their preferences, i.e., to change their evaluation concerning some government(s) or/and the importance of the criteria, in order to obtain a better political consensus.
\end{abstract}

Keywords Consensus reaching · Consensus degree $\cdot$ Government $\cdot$ Coalition · Policy

\section{Introduction}

In the literature, one may find many works on coalition formation theory; see, for instance, Austen-Smith and Banks (1988), Axelrod (1970), Baron (1993), De Swaan (1973), De Vries (1999), Grofman (1982), Kahan and Rapoport (1984), Kirchsteiger and Puppe (1997), Laver and Schofield (1990), Laver and Shepsle (1990, 1996), McKelvey et al. (1978), Peleg (1981), Schofield (1993a, 1993b, 1995), Shepsle (1979), Van Deemen (1991, 1997). An alternative model of multi-dimensional coalition formation has recently been presented in Rusinowska

\footnotetext{
P. Eklund

Department of Computing Science, Umeå University, 90187 Umeå, Sweden e-mail: peklund@cs.umu.se
}

\author{
A. Rusinowska ( $₫)$ \\ GATE - Groupe d'Analyse et de Théorie Economique, CNRS - Université Lumière Lyon 2, \\ 93 Chemin des Mouilles, B.P.167, 69131 Ecully Cedex, France \\ e-mail: rusinowska@gate.cnrs.fr \\ H. de Swart \\ Faculty of Philosophy, Tilburg University, P.O. Box 90153, 5000 LE Tilburg, The Netherlands \\ e-mail: H.C.M.deSwart@uvt.nl
}


et al. (2005). The central notion of this model is the notion of a stable government, where a government is defined as a pair consisting of a winning coalition and a policy supported by this coalition. A policy is a tuple of policies on given issues. In Rusinowska et al. (2005), necessary and sufficient conditions for the existence and uniqueness of a stable government are investigated. In this coalition formation model parties are supposed to have preferences regarding each winning coalition and regarding each policy on the given issues. These preferences are supposed to be constant and no possibility of adjusting the preferences of a party is considered.

A consensus model is analyzed in Carlsson et al. (1992), where the authors study the problem of formalizing consensus, within a set of decision makers trying to agree on a mutual decision. Convergence to consensus depends on the decision makers' willingness to compromise. Contrary to the model proposed in Rusinowska et al. (2005), in Carlsson et al. (1992), decision makers are often advised to adjust their preferences in order to obtain a better consensus.

The aim of this paper is to introduce a dynamic model of coalition formation in which parties may compromise in order to reach consensus. By combining some notions of both the consensus model (Carlsson et al. 1992) and the model of a stable government (Rusinowska et al. 2005), a new consensus model of political decision-making is constructed.

The paper is organized as follows. In Sect. 2, a model of coalition formation, and a procedure for consensus reaching within a coalition is presented. Each coalition 'entitled' to form a government tries to reach consensus on a government policy. We also discuss the stability property. In Sect. 3, we consider a procedure for choosing a feasible government from among all governments proposed by the coalitions. In Sect. 4, we conclude. The paper contains two appendices. In Appendix 1, we present some procedures different from the one proposed in Sect. 3. Appendix 2 presents an example illustrating the notions of our model of political consensus.

\section{Consensus reaching within a coalition}

\subsection{Preliminaries}

Let $N$ be the set of all parties in parliament, where $N=\{1, \ldots, n\}$. Let $W$ denote the set of all winning coalitions, that is,

$$
W=\left\{T \subseteq N \mid \sum_{i \in T} w_{i} \geq q\right\},
$$

where $w_{i}$ is the number of seats received by party $i$, and $q$ is the quota, i.e., the number of seats needed for a coalition to be a winning coalition. We assume that the winning coalitions are 'entitled' to form a government.

An important property of a winning coalition is the acceptability. We assume that each party $i \in N$ either accepts or does not accept a winning coalition it belongs to. Let $W_{i}$ be the set of all winning coalitions containing party $i \in N$ that are acceptable to party $i$.

The model concerns the creation of a government. It is assumed that there are some independent policy issues on which a government has to decide. Let $P$ be the set of all policies. A policy is said to be acceptable to party $i \in N$ if it is acceptable to this party with respect to each issue. Let $P_{i}$ denote the set of all policies acceptable to party $i \in N$. 
A government is defined as a pair $g=(S, p)$, where $S$ is a winning coalition and $p$ is a policy. Let $G$ denote the set of all governments. We then have

$$
G=\{(S, p) \mid S \in W \wedge p \in P\} .
$$

Next, the notion of feasible government is introduced. A government $(S, p)$ is feasible if both $S$ and $p$ are acceptable to each party belonging to $S$. Hence, the set $G^{*}$ of all feasible governments is equal to

$$
G^{*}=\left\{(S, p) \in G \mid \forall i \in S\left[S \in W_{i} \wedge p \in P_{i}\right]\right\}
$$

In this model, only feasible governments are considered. We introduce the set of all feasible coalitions as

$$
W^{*}=\left\{S \in W \mid \exists p \in P\left[(S, p) \in G^{*}\right]\right\} .
$$

Let $G_{i}^{*}$ be the set of all feasible governments containing party $i$, that is, for each $i \in N$,

$$
G_{i}^{*}=\left\{(S, p) \in G^{*} \mid i \in S\right\} .
$$

Of course, it may happen that $G_{i}^{*}=\emptyset$ for some $i \in N$.

A decision maker is a party $i \in N$ such that $G_{i}^{*} \neq \emptyset$. Let $D M$ denote the set of all decision makers, i.e.,

$$
D M=\left\{i \in N \mid G_{i}^{*} \neq \emptyset\right\} .
$$

A feasible government is evaluated by each member of this government with respect to the given policy issues and with respect to the issue concerning the coalition. Let $X$ be the finite set of criteria.

First of all, each decision maker evaluates the importance of the criteria. It may happen that one criterion, for instance, the winning coalition, is more important to a party than another one, i.e., than a certain policy issue. This means that it is more important to a given party which parties will form the government than which policy will be supported by this government. For each $i \in D M$, we assume $\alpha_{i}: X \rightarrow[0,1]$, such that

$$
\forall i \in D M\left[\sum_{x \in X} \alpha_{i}(x)=1\right] .
$$

$\alpha_{i}(x)$ is $i$ 's evaluation of criterion $x$. One way to determine the $\alpha_{i}$ in practice is by using the MACBETH software (Measuring Attractiveness by a Categorical Based Evaluation Technique). This is an interactive approach to quantify the attractiveness of each alternative, such that the measurement scale constructed is an interval scale; see Bana e Costa and Vansnick (1999), Bana e Costa et al. (2003), and the web site www.m-macbeth.com. The MACBETH software checks the consistency of the initial evaluations, and in case of any inconsistency it indicates to the user what is the cause of the inconsistency and how to improve on it in order to reach consistency.

Parties from a feasible coalition try to reach consensus. Let $G_{S}^{*}$ denote the set of all feasible governments formed by coalition $S \in W^{*}$. We then have

$$
G^{*}=\bigcup_{S \in W^{*}} G_{S}^{*} .
$$


Since all governments from $G_{S}^{*}$ are formed by coalition $S$, one may identify $G_{S}^{*}$ with the set of all policies acceptable to all parties from $S$.

Reaching consensus within a coalition means that the preferences of the parties from this coalition, as well as their evaluation of the importance of all criteria from $X$, should be relatively close to each other. When parties from a coalition reach consensus on a government involving this coalition, they may be confronted with governments formed by other coalitions, in which case the evaluation of the criterion 'winning coalition' may be quite important.

Given a feasible coalition $S \in W^{*}$, each party $i \in S$ evaluates each government from $G_{S}^{*}$ with respect to all the criteria. Hence, for each $i \in S$, we assume $f_{i, S}: X \times G_{S}^{*} \rightarrow[0,1]$ such that

$$
\forall x \in X\left[\sum_{y \in G_{S}^{*}} f_{i, S}(x, y)=1\right] .
$$

The value $f_{i, S}(x, y)$ is the value of government $y \in G_{S}^{*}$ to party $i \in D M$ with respect to criterion $x \in X$. Again, in practice, given party $i$ and criterion $x$ the values of $f_{i, S}(x, y)$ for the different governments $y \in G_{S}^{*}$ can be determined realistically by the MACBETH software.

Moreover, for each $i \in S$, we define $\beta_{i, S}: G_{S}^{*} \rightarrow[0,1]$ such that

$$
\left(\beta_{i, S}(y)\right)_{y \in G_{S}^{*}}=\left(\alpha_{i}(x)\right)_{x \in X} \cdot\left(f_{i, S}(x, y)\right)_{x \in X, y \in G_{S}^{*}},
$$

where $\left(\alpha_{i}(x)\right)_{x \in X}$ is the $1 \times|X|$ matrix representing the evaluation (comparison) of the criteria by party $i,\left(f_{i, S}(x, y)\right)_{x \in X, y \in G_{S}^{*}}$ is the $|X| \times\left|G_{S}^{*}\right|$ matrix containing party $i$ 's evaluation (comparison) of all governments in $G_{S}^{*}$ with respect to each criterion in $X$, and $\left(\beta_{i, S}(y)\right)_{y \in G_{S}^{*}}$ is the 'output', i.e., $1 \times\left|G_{S}^{*}\right|$ matrix containing party $i$ 's evaluation of each government in $G_{S}^{*}$. Because of (7) and (9),

$$
\sum_{y \in G_{S}^{*}} \beta_{i, S}(y)=1 \text {. }
$$

\subsection{Consensus degree}

Let $L G_{S}^{*}$ denote the set of all mappings $\beta_{i, S}: G_{S}^{*} \rightarrow[0,1]$. We define an assessment function $d: L G_{S}^{*} \times L G_{S}^{*} \rightarrow[0,1]$ satisfying the following two conditions:

(i) $d\left(\beta_{i, S}, \beta_{i, S}\right)=0$ for $i \in S$

(ii) $d\left(\beta_{i, S}, \beta_{j, S}\right)=d\left(\beta_{j, S}, \beta_{i, S}\right)$ for $i, j \in S$.

We call $\delta\left(\beta_{i, S}, \beta_{j, S}\right)=1-d\left(\beta_{i, S}, \beta_{j, S}\right)$ the consensus degree between decision makers $i$ and $j$ in coalition $S$. We may define $d\left(\beta_{i, S}, \beta_{j, S}\right)$ by a Euclidean-like 'distance' between the corresponding vectors. The higher the consensus (degree), the smaller the 'distance' between pairs of decision makers, i.e., between $\beta_{i, S}$ and $\beta_{j, S}$. In particular, if $d\left(\beta_{i, S}, \beta_{j, S}\right)=0$ (i.e., $\delta\left(\beta_{i, S}, \beta_{j, S}\right)=1$ ), then we say that $i$ and $j$ are in complete consensus in coalition $S$. If $d\left(\beta_{i, S}, \beta_{j, S}\right)=1$ (i.e., $\left.\delta\left(\beta_{i, S}, \beta_{j, S}\right)=0\right)$, then we say that $i$ and $j$ are in complete disagreement in coalition $S$. Let $i, j \in S$. We propose the Euclidean-like 'distance' given by

$$
d\left(\beta_{i, S}, \beta_{j, S}\right)=\sqrt{\frac{1}{\left|G_{S}^{*}\right|} \sum_{y \in G_{S}^{*}}\left(\beta_{i, S}(y)-\beta_{j, S}(y)\right)^{2}},
$$


where $\left|G_{S}^{*}\right|$ denotes the number of alternative governments for coalition $S$.

Next, we define

$$
d_{S}^{*}=\max \left\{d\left(\beta_{i, S}, \beta_{j, S}\right) \mid \beta_{i, S}, \beta_{j, S} \in L G_{S}^{*}\right\},
$$

and a generalized consensus degree for coalition $S$ as

$$
\delta_{S}^{*}=1-d_{S}^{*}
$$

The generalized consensus degree $\delta_{S}^{*}$ concerns the consensus reached by all the decision makers from coalition $S$.

\subsection{Consensus reaching}

In this sub-section we specify a procedure for consensus reaching within a coalition. If parties are sufficiently willing to compromise, the procedure will result in reaching consensus, and in proposing one feasible government formed by the given coalition. A certain consensus degree $0<\widetilde{\delta}<1$ is required in the model. We say that coalition $S$ reaches consensus if the generalized consensus degree $\delta_{S}^{*}$ is not smaller than $\tilde{\delta}$, that is, if $\delta_{S}^{*} \geq \tilde{\delta}$. If $\delta_{S}^{*}<\tilde{\delta}$, then the parties do not reach consensus, of course, if (some of) these parties do not adjust their preferences.

Note that, in particular, if $G_{S}^{*}=\{y\}$, then for each $i \in S$, and for each $x \in X, f_{i, S}(x, y)=$ 1 , and hence, for each $i \in S, \beta_{i, S}(y)=1$. So, in this case, $\delta_{S}^{*}=1$. This means that if there is only one alternative to a given coalition $S$, this coalition will reach (complete) consensus.

Let $D_{S}^{*}$ be the set of parties from $S$ with most different preferences, that is,

$$
D_{S}^{*}=\left\{i \in S \mid \exists j \in S\left[d\left(\beta_{i, S}, \beta_{j, S}\right)=d_{S}^{*}\right]\right\} .
$$

For each feasible coalition, we assume a kind of mediator, called the chairman. The chairman does not belong to any party and he is indifferent between all the parties. The chairman will decide which party from $D_{S}^{*}$ will be advised to change its preferences (i.e., evaluations) regarding some government(s) or/and the importance of the criteria. It seems reasonable to assume that a party asked to adjust its preferences is a party $i_{S}^{D} \in D_{S}^{*}$ such that

$$
i_{S}^{D}=\arg \max _{i \in D_{S}^{*}} \sum_{j \in S} d\left(\beta_{i, S}, \beta_{j, S}\right) .
$$

If there are at least two such parties, the chairman chooses one of them. Moreover, the chairman proposes such a change to party $i_{S}^{D}$ that the consensus degree will increase if this party follows the chairman's advice. If possible, the chairman's advice should lead to a new consensus degree not smaller than $\widetilde{\delta}$. If the party does not agree to adjust its preferences (evaluations) according to the chairman's advice, the chairman may propose another change to the same party or a change to another party. If consensus is reached for a given coalition $S$, that is, if the generalized (final) consensus degree is not smaller than $\widetilde{\delta}$, a consensus decision for coalition $S$ is determined as follows. We add up the weighted (final) values of the alternatives to all decision makers from $S$. For each $y \in G_{S}^{*}$, the weighted value $\beta_{S}(y)$ of alternative $y$ to coalition $S$ is defined as

$$
\beta_{S}(y)=\sum_{i \in S} w_{i}^{\prime} \cdot \beta_{i, S}(y),
$$


where for each $i \in S$

$$
w_{i}^{\prime}=\frac{w_{i}}{\sum_{j \in S} w_{j}} .
$$

For calculating the $\beta_{S}$ 's we decided for the weighted sum, but we could also treat all the parties equally, and define $\beta_{S}(y)$ for each $y \in G_{S}^{*}$ as $\beta_{S}(y)=\sum_{i \in S} \beta_{i, S}(y)$.

Coalition $S$ chooses the government $y_{S}^{*}$ such that

$$
y_{S}^{*}=\arg \max _{y \in G_{S}^{*}} \beta_{S}(y) .
$$

If there are two such governments, the chairman chooses one of them.

Generally, if in the chairman's opinion it does not make any sense to continue the attempts to reach consensus within the coalition $S$, the decision making about forming a government by $S$ is postponed, and the given coalition is involved in no government. This means that the given coalition does not propose any government to be formed.

\subsection{Stability}

Let $Y$ be a set of alternatives, and let $D M$ be the set of decision makers involved in them. We assume that for each $i \in D M$, there is a function $\beta_{i}: Y \rightarrow[0,1]$. In practice, the values of the $\beta_{i}(y)$ 's for the different $y \in Y$ can be determined realistically by the MACBETH decision support system.

We say that alternative $y^{\prime} \in Y$ dominates alternative $y \in Y$ in $S \subseteq D M\left(y^{\prime} \succ_{S} y\right)$ if

$$
\forall i \in S\left[\beta_{i}\left(y^{\prime}\right) \geq \beta_{i}(y)\right] \wedge \exists i \in S\left[\beta_{i}\left(y^{\prime}\right)>\beta_{i}(y)\right] .
$$

Moreover, we say that alternative $y \in Y$ is stable in $S$ with respect to $Y$ if there is no alternative in $Y$ dominating $y$ in $S$, that is, if

$$
\forall y^{\prime} \in Y\left[\neg\left(y^{\prime} \succ_{S} y\right)\right] .
$$

From the definition of $y_{S}^{*}$ the following remark is evident.

Remark 1 If $y_{S}^{*}$ is the government chosen by consensus reaching within coalition $S$, then $y_{S}^{*}$ is stable in $S$ with respect to $G_{S}^{*}$.

\section{Choosing a government}

Each feasible coalition $S \in W^{*}$ in which the parties reach consensus, proposes the government $y_{S}^{*}$ agreed upon. If there is only one feasible government proposed, i.e., if only one coalition managed to reach consensus, this government is chosen, and finally formed. If there is no feasible government proposed, of course, no government is formed.

Suppose that at least two feasible governments are proposed, which means that at least two coalitions succeeded in reaching consensus. Let $Y^{*}$ be the set of all the governments resulting from consensus reached by feasible coalitions, where $1<\left|Y^{*}\right| \leq\left|W^{*}\right|$. Let $D M^{*}$ be the set of all the parties involved in at least one consensus government, i.e.,

$$
D M^{*}=\left\{i \in D M \mid \exists S \in W^{*}\left[i \in S \wedge y_{S}^{*} \in Y^{*}\right]\right\} .
$$


Let $Y_{i}^{*}$ denote the set of all governments from $Y^{*}$ containing party $i \in D M^{*}$, i.e., for each $i \in D M^{*}$

$$
Y_{i}^{*}=\left\{y_{S}^{*} \in Y^{*} \mid i \in S\right\} .
$$

Of course, for each $i \in D M^{*}, Y_{i}^{*} \neq \emptyset$.

Each party $i \in D M^{*}$ evaluates each government from $Y_{i}^{*}$ with respect to all the criteria from $X$. Hence, for each $i \in D M^{*}$, there is $f_{i}: X \times Y_{i}^{*} \rightarrow[0,1]$ such that

$$
\forall x \in X\left[\sum_{y \in Y_{i}^{*}} f_{i}(x, y)=1\right] .
$$

Again, one may use the MACBETH software in order to determine the values of $f_{i}(x, y)$ for the different $y \in Y_{i}^{*}$, given party $i$ and criterion $x$.

For each $i \in D M^{*}, \beta_{i}: Y_{i}^{*} \rightarrow[0,1]$ is defined by

$$
\left(\beta_{i}(y)\right)_{y \in Y_{i}^{*}}=\left(\alpha_{i}(x)\right)_{x \in X} \cdot\left(f_{i}(x, y)\right)_{x \in X, y \in Y_{i}^{*}} .
$$

In order to avoid some 'dominated' solutions, we add a condition expressing a kind of 'internal stability'. Since we do not restrict feasible coalitions to minimal winning coalitions, it may happen that there are two coalitions from $W^{*}$ such that one of them contains the other. Let us consider the following condition.

We say that a government $y^{*}=\left(S^{*}, p^{*}\right) \in Y^{*}$ satisfies internal stability (or, is internally stable), if

$$
\begin{aligned}
& \neg \exists(S, p) \in Y^{*}\left[\left(S \subset S^{*} \vee S^{*} \subset S\right)\right. \\
& \left.\quad \wedge \forall i \in S \cap S^{*}\left[\beta_{i}(S, p) \geq \beta_{i}\left(y^{*}\right)\right] \wedge \exists i \in S \cap S^{*}\left[\beta_{i}(S, p)>\beta_{i}\left(y^{*}\right)\right]\right] .
\end{aligned}
$$

Condition (26) says that there is no subset $S$ of coalition $S^{*}$, and $S^{*}$ is a subset of no coalition $S$ that can form its own government $y=(S, p) \in Y^{*}$ such that all parties from $S \cap S^{*}$ evaluate $y$ at least as high as $y^{*}$, and at least one party from $S \cap S^{*}$ evaluates $y$ higher than $y^{*}$. If this condition were not satisfied, then the parties from coalition $S \cap S^{*}$ would resign from $y^{*}$ and form the government $y$. Let $Y^{* *}$ be the set of all internally stable governments, i.e.,

$$
Y^{* *}=\left\{y^{*} \in Y^{*} \mid y^{*} \text { is internally stable }\right\} .
$$

If there is only one government from $Y^{* *}$, this government is chosen. Suppose now that there are at least two such governments, i.e., $\left|Y^{* *}\right|>1$.

Let $Y_{i}^{* *}$ be the set of all internally stable governments containing party $i \in D M^{*}$, i.e.,

$$
Y_{i}^{* *}=\left\{y_{S}^{*} \in Y^{* *} \mid i \in S\right\} .
$$

Let $W^{* *}$ be the set of all the feasible coalitions forming an internally stable government, i.e.,

$$
W^{* *}=\left\{S \in W^{*} \mid y_{S}^{*} \in Y^{* *}\right\},
$$

and let $D M^{* *}$ denote the set of all decision makers involved in at least one internally stable government, i.e.,

$$
D M^{* *}=\left\{i \in D M^{*} \mid \exists S \in W^{* *}[i \in S]\right\} .
$$


In order to choose a government from $Y^{* *}$ in case $\left|Y^{* *}\right|>1$, we propose a procedure in which the coalition with the highest consensus is awarded, and is asked to form a government. The government chosen is $y^{(0)}=\left(S^{(0)}, p^{(0)}\right) \in Y^{* *}$ satisfying the following condition

$$
S^{(0)}=\arg \max _{S \in W^{* *}} \delta_{S}^{*} .
$$

Suppose that there are at least two solutions of this condition. We may then choose the coalition in which two players are most 'close' to each other, or, alternatively, the coalition with the minimal average distance. Let for each $S \in W^{* *}$

$$
c_{S}^{*}=\min \left\{d\left(\beta_{i, S}, \beta_{j, S}\right) \mid i, j \in S\right\} .
$$

Moreover, let $W_{(0)}^{* *}$ denote the set of all the solutions of condition (31). Then, the government chosen by this procedure is a government $y^{(0)}=\left(S^{(0)}, p^{(0)}\right) \in Y^{* *}$ satisfying the extra condition

$$
S^{(0)}=\arg \min _{S \in W_{(0)}^{* *}} c_{S}^{*} .
$$

In the unlikely case that there are at least two solutions of condition (33), one may apply other procedures. We discuss some of them in Appendix 1.

\section{Conclusions}

In this paper, a consensus model for coalition formation has been proposed. If parties are willing to compromise, it is always possible to reach consensus, and to create a feasible government. In the procedure there is an 'outsider', called the chairman, who advises parties how to adjust their preferences. Clearly, the consensus agreed upon depends on the suggestions of the chairman. First, each feasible coalition tries to reach consensus within this coalition about the government to be formed. Parties consider only feasible governments, i.e., governments acceptable for all parties belonging to the coalition involved, and if there is only one feasible government they can form, they agree. If the parties from a given coalition manage to reach consensus, the coalition proposes to form the government agreed upon. This consensus government is stable in the given coalition with respect to the set of all feasible governments formed by that coalition.

It may happen, of course, that no feasible coalition reaches consensus. In this case, no final government is created. If there is only one feasible coalition which reaches consensus, then the government proposed by this coalition is formed. If there are at least two coalitions that succeed in reaching consensus, that is, if at least two governments are proposed, we select the governments which are 'internally stable'. Next, if there are at least two such governments, an extra procedure is applied in order to choose one of these governments. Essentially, we propose one such procedure, but some alternative procedures for choosing a government are considered in the appendix.

The model presented in this paper may be extended by incorporating portfolio distribution issues into the model. Since a portfolio distribution depends on a governing coalition, it seems proper to treat 'portfolio distribution' as an additional issue dependent on 'winning coalition'. Hence, instead of considering (winning) coalitions only, one may analyze an independent issue consisting of two dependent sub-issues: 'winning coalition' and 'portfolio distribution'. 
The consensus model can also be applied to choosing an alternative from a set of alternatives by a committee.

The protocol given in this paper can be automatized, resulting in a decision support system for coalition-government formation. The informational requirements of the proposed protocol are demanding, but the MACBETH software can deliver all information needed in a very rational and perspicuous way; see Roubens et al. (2006).

Acknowledgements The authors wish to thank gratefully Professor Hannu Nurmi and two anonymous referees for many useful suggestions concerning this paper. A. Rusinowska gratefully acknowledges support by COST Action 274, TARSKI.

\section{Appendix 1: Different procedures of choosing a government}

Procedures focused on one party The main feature of these procedures is that one party from $D M^{* *}$ is asked to form a government. Let us denote this chosen party by $i^{*}$. Party $i^{*}$ is appointed by a kind of chairman or supervisor (for instance, the Queen in the Netherlands). Most likely, $i^{*}$ will be the strongest party, i.e., the party with the greatest number of seats in parliament, but we leave it open. In principle, $i^{*}$ may be each party from $D M^{* *}$.

In a procedure, which we call quick procedure, party $i^{*}$ simply chooses its best government (if there are more than one, $i^{*}$ chooses one of them). Denoting by $y^{(1)}=\left(S^{(1)}, p^{(1)}\right) \in$ $Y^{* *}$ the government chosen by using this procedure, we get

$$
y^{(1)}=\arg \max _{y \in Y_{i^{*}}^{* *}} \beta_{i^{*}}(y) .
$$

Another procedure is a procedure based on negotiations. This procedure is also based on party $i^{*}$, but is not necessarily so advantageous to $i^{*}$. We will denote the government resulting from applying this procedure by $y^{(2)} \in Y^{* *}$. In this procedure, party $i^{*}$ is also asked to form a government, but $i^{*}$ 's choice of one government is not sufficient to create it. Forming a final government requires the approval of all parties belonging to the given feasible coalition. In this case, the parties are involved in some very simple negotiations. Party $i^{*}$ proposes to form its best government $y^{(1)}=\left(S^{(1)}, p^{(1)}\right)$ defined in (34), and each party from $S^{(1)}$ has to react to this offer. A party from $S^{(1)}$ either agrees or disagrees. The government $y^{(1)}$ is formed, that is, $y^{(2)}=y^{(1)}$, if all parties from $S^{(1)}$ accept the offer.

If at least one party from $S^{(1)}$ disagrees to form the government $y^{(1)}$, party $i^{*}$ switches to another coalition from $W^{* *}$ containing party $i^{*}$, the one which forms the second best government from $Y_{i^{*}}^{* *}$ to party $i^{*}$, etc. We assume that the parties always behave rationally, and that they prefer any government formed to no government formed. Then, creating no government from the set $Y_{i^{*}}^{* *}$ by party $i^{*}$ means that there is at least one government from $Y^{* *}$ not containing party $i^{*}$, i.e., $Y^{* *} \backslash Y_{i^{*}}^{* *} \neq \emptyset$. In this case, our chairman appoints another party to continue forming a final government. This new 'leader' has to be involved in at least one government from the set $Y^{* *} \backslash Y_{i^{*}}^{* *}$, and it may (but does not have to) be the strongest party involved in a government from $Y^{* *} \backslash Y_{i^{*}}^{* *}$. This new party, let us say, party $j^{*}$, evaluates all the governments from the set $Y_{j^{*}}^{* *} \cap\left(Y^{* *} \backslash Y_{i^{*}}^{* *}\right)$, and it proposes to form the one which is best to party $j^{*}$, etc. We repeat the procedure as long as the final government has not been chosen.

The procedure based on negotiations is now being used both in Finland and in the Netherlands, where the strongest party is asked to form a government. If this party turns out to be not successful, another party is asked to form a government. 
Procedure based on total gain One may also consider a procedure which gives a result maximizing the total value for all parties from $D M^{* *}$. As was suggested before, let us assume now that each party $i \in D M^{* *}$ has an evaluation of each government from the set $Y^{* *}$ with respect to all the criteria from $X$, whether the party belongs to this government or not. Hence, for each $i \in D M^{* *}$, we assume $F_{i}: X \times Y^{* *} \rightarrow[0,1]$ such that

$$
\forall x \in X\left[\sum_{y \in Y^{* *}} F_{i}(x, y)=1\right],
$$

and define $B_{i}: Y^{* *} \rightarrow[0,1]$ by

$$
\left(B_{i}(y)\right)_{y \in Y^{* *}}=\left(\alpha_{i}(x)\right)_{x \in X} \cdot\left(F_{i}(x, y)\right)_{x \in X, y \in Y^{* *}} .
$$

Again, one may use the MacBeth software to determine the values of $F_{i}(x, y)$. The government chosen by using this procedure is a government $y^{(3)}=\left(S^{(3)}, p^{(3)}\right) \in Y^{* *}$ satisfying the following condition

$$
y^{(3)}=\arg \max _{y \in Y^{* *}} \sum_{i \in D M^{* *}} W_{i}^{\prime} \cdot B_{i}(y),
$$

where for each $i \in D M^{* *}$

$$
W_{i}^{\prime}=\frac{w_{i}}{\sum_{j \in D M^{* *}} w_{j}} .
$$

If there is more than one government satisfying this condition, some extra method(s) may be applied. Suppose that, for instance, there are two governments satisfying this condition, but the majority of the decision makers prefers one of them. Then, this government preferred by the majority may be chosen.

Condition (37) says that the government $y^{(3)}$ maximizes the weighted sum of the values $B_{i}(y)$ of all decision makers $i$ from $D M^{* *}$. Of course, this does not mean that the government is the best for the parties forming this government. This chosen government may be, for instance, more popular among the parties not belonging to this government, but less advantageous to its members.

\section{Appendix 2: Example}

In order to illustrate the main procedure for consensus reaching (described in Sects. 2 and 3), let us analyze the following simple example. We consider a parliament consisting of five parties,

$$
N=\{A, B, C, D, E\},
$$

with the quota $q=51$, and the following weights of the parties:

$$
w_{A}=30, \quad w_{B}=20, \quad w_{C}=17, \quad w_{D}=18, \quad w_{E}=15 .
$$

Hence, there are 15 winning coalitions, but not all these coalitions are acceptable to their members. Neither party $A$ nor $B$ does accept any coalition with $E$. Party $E$ in its turn does not accept any coalition either with $A$ or with $B$. Moreover, party $C$ does not accept any coalition with $D$, and $D$ does not accept any coalition with $C$ (see Myerson 1977 on the 
willingness to cooperate). Hence, we have only two winning coalitions acceptable to all their members, that is, coalition $A B C$ and $A B D$. Using the notation from Sect. 2, we have

$$
\begin{aligned}
& W_{A}=\{A B C, A B D\}, \quad W_{B}=\{A B C, A B D\} \\
& W_{C}=\{A B C\}, \quad W_{D}=\{A B D\}, \quad W_{E}=\emptyset .
\end{aligned}
$$

Moreover, suppose there are four policies, i.e.,

$$
P=\left\{p_{1}, p_{2}, p_{3}, p_{4}\right\}
$$

with respect to two policy issues $x_{1}$ and $x_{2}$. Hence, the set of all criteria is equal to

$$
X=\left\{x_{1}, x_{2}, x_{3}\right\}
$$

where criterion $x_{3}$ concerns the winning coalition. Assume that both parties $A$ and $B$ accept all four policies, party $C$ does not accept policy $p_{4}$, and $D$ does not accept policy $p_{3}$. Hence, we have

$$
P_{A}=P_{B}=P, \quad P_{C}=\left\{p_{1}, p_{2}, p_{3}\right\}, \quad P_{D}=\left\{p_{1}, p_{2}, p_{4}\right\} .
$$

The set of all feasible governments is then equal to

$$
G^{*}=\left\{y_{1}, y_{2}, y_{3}, y_{4}, y_{5}, y_{6}\right\},
$$

where

$$
\begin{array}{lll}
y_{1}=\left(A B C, p_{1}\right), & y_{2}=\left(A B C, p_{2}\right), & y_{3}=\left(A B C, p_{3}\right) \\
y_{4}=\left(A B D, p_{1}\right), & y_{5}=\left(A B D, p_{2}\right), & y_{6}=\left(A B D, p_{4}\right) .
\end{array}
$$

Moreover, using the notation from Sect. 2, we have

$$
\begin{aligned}
& G_{A}^{*}=G_{B}^{*}=G^{*}, \quad G_{C}^{*}=\left\{y_{1}, y_{2}, y_{3}\right\}, \quad G_{D}^{*}=\left\{y_{4}, y_{5}, y_{6}\right\}, \quad G_{E}^{*}=\emptyset \\
& G_{A B C}^{*}=\left\{y_{1}, y_{2}, y_{3}\right\}, \quad G_{A B D}^{*}=\left\{y_{4}, y_{5}, y_{6}\right\} .
\end{aligned}
$$

The set of all decision makers $D M$, and the set of all feasible coalitions $W^{*}$ are equal to

$$
D M=\{A, B, C, D\}, \quad W^{*}=\{A B C, A B D\} .
$$

We assume the required consensus degree $\widetilde{\delta}=0.85$.

The parties' evaluations of the importance of the criteria are as follows:

$$
\begin{array}{ll}
\alpha_{A}=\left(\frac{1}{3}, \frac{1}{3}, \frac{1}{3}\right), & \alpha_{B}=\left(\frac{1}{2}, \frac{1}{4}, \frac{1}{4}\right), \\
\alpha_{C}=\left(\frac{1}{4}, \frac{1}{4}, \frac{1}{2}\right), & \alpha_{D}=\left(\frac{1}{6}, \frac{1}{3}, \frac{1}{2}\right) .
\end{array}
$$

Moreover, the comparison matrices for the parties are the following:

$$
\left(f_{A, A B C}(x, y)\right)_{x \in X, y \in G_{A B C}^{*}}=\left(\begin{array}{ccc}
\frac{1}{3} & \frac{1}{6} & \frac{1}{2} \\
\frac{1}{3} & \frac{1}{6} & \frac{1}{2} \\
\frac{1}{3} & \frac{1}{3} & \frac{1}{3}
\end{array}\right),
$$




$$
\begin{aligned}
\left(f_{B, A B C}(x, y)\right)_{x \in X, y \in G_{A B C}^{*}} & =\left(\begin{array}{ccc}
\frac{1}{4} & \frac{1}{4} & \frac{1}{2} \\
\frac{1}{6} & \frac{1}{6} & \frac{2}{3} \\
\frac{1}{3} & \frac{1}{3} & \frac{1}{3}
\end{array}\right), \\
\left(f_{C, A B C}(x, y)\right)_{x \in X, y \in G_{A B C}^{*}} & =\left(\begin{array}{ccc}
\frac{1}{2} & \frac{1}{3} & \frac{1}{6} \\
\frac{1}{3} & \frac{1}{2} & \frac{1}{6} \\
\frac{1}{3} & \frac{1}{3} & \frac{1}{3}
\end{array}\right), \\
\left(f_{A, A B D}(x, y)\right)_{x \in X, y \in G_{A B D}^{*}} & =\left(\begin{array}{ccc}
\frac{1}{2} & \frac{1}{4} & \frac{1}{4} \\
\frac{1}{2} & \frac{1}{4} & \frac{1}{4} \\
\frac{1}{3} & \frac{1}{3} & \frac{1}{3}
\end{array}\right), \\
\left(f_{B, A B D}(x, y)\right)_{x \in X, y \in G_{A B D}^{*}} & =\left(\begin{array}{ccc}
\frac{1}{6} & \frac{1}{6} & \frac{2}{3} \\
\frac{1}{3} & \frac{1}{3} & \frac{1}{3} \\
\frac{1}{3} & \frac{1}{3} & \frac{1}{3}
\end{array}\right), \\
\left(f_{D, A B D}(x, y)\right)_{x \in X, y \in G_{A B D}^{*}} & =\left(\begin{array}{ccc}
\frac{1}{3} & \frac{1}{3} & \frac{1}{3} \\
\frac{1}{3} & \frac{1}{3} & \frac{1}{3} \\
\frac{1}{3} & \frac{1}{3} & \frac{1}{3}
\end{array}\right) .
\end{aligned}
$$

Hence, we get

$$
\begin{aligned}
& \left(\beta_{A, A B C}(y)\right)_{y \in G_{A B C}^{*}}=\left(\frac{3}{9}, \frac{2}{9}, \frac{4}{9}\right), \quad\left(\beta_{A, A B D}(y)\right)_{y \in G_{A B D}^{*}}=\left(\frac{8}{18}, \frac{5}{18}, \frac{5}{18}\right) \\
& \left(\beta_{B, A B C}(y)\right)_{y \in G_{A B C}^{*}}=\left(\beta_{B, A B D}(y)\right)_{y \in G_{A B D}^{*}}=\left(\frac{1}{4}, \frac{1}{4}, \frac{1}{2}\right) \\
& \left(\beta_{C, A B C}(y)\right)_{y \in G_{A B C}^{*}}=\left(\frac{3}{8}, \frac{3}{8}, \frac{2}{8}\right), \quad\left(\beta_{D, A B D}(y)\right)_{y \in G_{A B D}^{*}}=\left(\frac{1}{3}, \frac{1}{3}, \frac{1}{3}\right) .
\end{aligned}
$$

Consensus reaching within coalition $A B C$ Since $G_{A B C}^{*} \neq \emptyset$, parties $A, B$, and $C$ will try to reach consensus concerning the alternatives $y_{1}, y_{2}$, and $y_{3}$. We get

$$
\begin{aligned}
& 0.06<d\left(\beta_{A, A B C}, \beta_{B, A B C}\right)<0.07, \quad 0.14<d\left(\beta_{A, A B C}, \beta_{C, A B C}\right)<0.15 \\
& 0.17<d\left(\beta_{B, A B C}, \beta_{C, A B C}\right)<0.18 .
\end{aligned}
$$

Hence,

$$
d_{A B C}^{*}=d\left(\beta_{B, A B C}, \beta_{C, A B C}\right)>0.17,
$$

and hence, the generalized consensus degree for coalition $A B C$ is

$$
\delta_{A B C}^{*}=1-d_{A B C}^{*}<0.83<0.85=\tilde{\delta} .
$$

Moreover,

$$
D_{A B C}^{*}=\{B, C\},
$$

which means that either $B$ or $C$ will be asked by the chairman to adjust its preferences. We check which party will be appointed as party $i_{A B C}^{D}$. Since the distance between $A$ and $C$ is 
greater than the distance between $A$ and $B$, we have

$$
\sum_{j \in A B C} d\left(\beta_{C, A B C}, \beta_{j, A B C}\right)>\sum_{j \in A B C} d\left(\beta_{B, A B C}, \beta_{j, A B C}\right),
$$

and hence $i_{A B C}^{D}=C$. This means that party $C$ will be advised to adjust its preferences. Let us suppose that the chairman advises party $C$ to change its evaluation of the governments with respect to the first criterion, $x_{1}$. Let the new preferences of $C$ be as follows

$$
\left(f_{C, A B C}^{\prime}(x, y)\right)_{x \in X, y \in G_{A B C}^{*}}=\left(\begin{array}{ccc}
\frac{1}{3} & \frac{1}{6} & \frac{1}{2} \\
\frac{1}{3} & \frac{1}{2} & \frac{1}{6} \\
\frac{1}{3} & \frac{1}{3} & \frac{1}{3}
\end{array}\right) .
$$

With respect to criterion $x_{1}$, both parties $A$ and $B$ like the third alternative $y_{3}$ most. Hence, if party $C$ changes its evaluation of the governments with respect to $x_{1}$ such that $y_{3}$ becomes the best alternative to $C$, party $C$ 's preferences will become 'closer' to the preferences of parties $A$ and $B$. Suppose that party $C$ agrees to follow the advice of the chairman. Hence, the new values are equal to

$$
\left(\beta_{C, A B C}^{\prime}(y)\right)_{y \in G_{A B C}^{*}}=\left(\frac{1}{3}, \frac{1}{3}, \frac{1}{3}\right),
$$

and hence we get

$$
\begin{aligned}
& d\left(\beta_{A, A B C}^{\prime}, \beta_{B, A B C}^{\prime}\right)=d\left(\beta_{A, A B C}, \beta_{B, A B C}\right)<0.07, \\
& 0.09<d\left(\beta_{A, A B C}^{\prime}, \beta_{C, A B C}^{\prime}\right)<0.1, \\
& 0.11<d\left(\beta_{B, A B C}^{\prime}, \beta_{C, A B C}^{\prime}\right)<0.12 .
\end{aligned}
$$

Hence, now,

$$
d_{A B C}^{*}=d\left(\beta_{B, A B C}^{\prime}, \beta_{C, A B C}^{\prime}\right)<0.12,
$$

and consequently,

$$
\delta_{A B C}^{*}>0.88>0.85=\widetilde{\delta} .
$$

This means, of course, that parties $A, B$, and $C$, reach consensus. In order to find a consensus decision, we calculate the following values

$$
w_{A}^{\prime}=\frac{30}{67}, \quad w_{B}^{\prime}=\frac{20}{67}, \quad w_{C}^{\prime}=\frac{17}{67} .
$$

Moreover, for $k=1,2,3$, we calculate

$$
\beta_{A B C}\left(y_{k}\right)=w_{A}^{\prime} \cdot \beta_{A, A B C}\left(y_{k}\right)+w_{B}^{\prime} \cdot \beta_{B, A B C}\left(y_{k}\right)+w_{C}^{\prime} \cdot \beta_{C, A B C}^{\prime}\left(y_{k}\right),
$$

and we get

$$
\beta_{A B C}\left(y_{1}\right)=\frac{62}{201}, \quad \beta_{A B C}\left(y_{2}\right)=\frac{52}{201}, \quad \beta_{A B C}\left(y_{3}\right)=\frac{87}{201} .
$$

This means that coalition $A B C$ proposes government $y_{3}$, i.e,

$$
y_{A B C}^{*}=y_{3} .
$$


Consensus reaching within coalition $A B D$ In a similar way, one may consider consensus reaching within coalition $A B D$, since $G_{A B D}^{*} \neq \emptyset$. Parties $A, B$, and $D$ will try to reach consensus concerning alternatives $y_{4}, y_{5}$, and $y_{6}$. Let us suppose that after consensus reaching within coalition $A B D$, the coalition proposes government $y_{4}$, i.e.,

$$
y_{A B D}^{*}=y_{4} .
$$

Choosing one government In our example, two governments are proposed: $y_{3}=\left(A B C, p_{3}\right)$ by coalition $A B C$, and $y_{4}=\left(A B D, p_{1}\right)$ by coalition $A B D$. Since $A B C \not \subset A B D$, and $A B D \not \subset$ $A B C$, both governments are internally stable. Using the notations from Sect. 3, we get

$$
\begin{aligned}
& Y^{* *}=Y^{*}=\left\{y_{3}, y_{4}\right\}, \quad W^{* *}=W^{*}=\{A B C, A B D\} \\
& D M^{* *}=D M^{*}=\{A, B, C, D\} .
\end{aligned}
$$

Hence, since $\left|Y^{* *}\right|=2>1$, we will apply to this example the procedure introduced in Sect. 3. Taking into account the parties preferences mentioned in the beginning of this example, we have the following

$$
\left(f_{A}(x, y)\right)_{x \in X, y \in Y_{A}^{*}}=\left(F_{A}(x, y)\right)_{x \in X, y \in Y^{* *}}=\left(\begin{array}{cc}
\frac{3}{5} & \frac{2}{5} \\
\frac{3}{5} & \frac{2}{5} \\
\frac{1}{3} & \frac{2}{3}
\end{array}\right) .
$$

This matrix is derived from matrix $M=\left(f_{A, A B C}(x, y)\right)_{x \in X, y \in G_{A B C}^{*}}$ as follows. The first row concerns the relative preferences of $A$ for the policy $p_{3}$ of $y_{3}$, and the policy $p_{1}$ of $y_{4}$ with respect to criterion $x_{1}$. The first row of matrix $M$ tells us that the preferences of $A$ for $p_{3}$ and $p_{1}$ with respect to criterion $x_{1}$ are proportional to $\frac{1}{2}: \frac{1}{3}$, which is proportional to $\frac{3}{5}: \frac{2}{5}$, where $\frac{3}{5}+\frac{2}{5}=1$. A similar argument gives the second row. The third row concerns the relative preferences of $A$ for $y_{3}$ and $y_{4}$ with respect to criterion $x_{3}$, i.e., with respect to the coalitions $A B C$ and $A B D$. In our matrix we have assumed that $A$ prefers the coalition $A B D$ twice as much as the coalition $A B C$.

$$
\begin{aligned}
& \left(f_{B}(x, y)\right)_{x \in X, y \in Y_{B}^{*}}=\left(F_{B}(x, y)\right)_{x \in X, y \in Y^{* *}}=\left(\begin{array}{cc}
\frac{2}{3} & \frac{1}{3} \\
\frac{4}{5} & \frac{1}{5} \\
\frac{2}{3} & \frac{1}{3}
\end{array}\right) \\
& \left(f_{C}(x, y)\right)_{x \in X, y \in Y_{C}^{*}}=\left(f_{D}(x, y)\right)_{x \in X, y \in Y_{D}^{*}}=\left(\begin{array}{c}
1 \\
1 \\
1
\end{array}\right) \\
& \left(F_{C}(x, y)\right)_{x \in X, y \in Y^{* *}}=\left(\begin{array}{cc}
\frac{3}{5} & \frac{2}{5} \\
\frac{1}{3} & \frac{2}{3} \\
1 & 0
\end{array}\right), \quad\left(F_{D}(x, y)\right)_{x \in X, y \in Y^{* *}}=\left(\begin{array}{cc}
0 & 1 \\
0 & 1 \\
0 & 1
\end{array}\right) .
\end{aligned}
$$

(Note that the matrix $F_{C}$ is derived from the matrix $f_{C, A B C}^{\prime}$ and not from $f_{C, A B C}$.) Hence,

$$
\begin{aligned}
& \left(\beta_{A}(y)\right)_{y \in Y_{A}^{*}}=\left(B_{A}(y)\right)_{y \in Y^{* *}}=\left(\frac{23}{45}, \frac{22}{45}\right) \\
& \left(\beta_{B}(y)\right)_{y \in Y_{B}^{*}}=\left(B_{B}(y)\right)_{y \in Y^{* *}}=\left(\frac{7}{10}, \frac{3}{10}\right), \quad\left(\beta_{C}\left(y_{3}\right)\right)=\left(\beta_{D}\left(y_{4}\right)\right)=1
\end{aligned}
$$




$$
\left(B_{C}(y)\right)_{y \in Y^{* *}}=\left(\frac{11}{15}, \frac{4}{15}\right), \quad\left(B_{D}(y)\right)_{y \in Y^{* *}}=(0,1) .
$$

In our example,

$$
\delta_{A B C}^{*}=\delta_{A B D}^{*}=1-\frac{1}{\sqrt{72}},
$$

and hence, we apply the 'closest' condition. We have

$$
\begin{aligned}
& c_{A B C}^{*}=d\left(\beta_{A, A B C}, \beta_{B, A B C}\right)=\frac{1}{36} \sqrt{\frac{14}{3}}>0.06 \\
& c_{A B D}^{*}=d\left(\beta_{A, A B D}^{\prime}, \beta_{B, A B D}^{\prime}\right)=\frac{\sqrt{2}}{36}<0.04,
\end{aligned}
$$

and therefore

$$
S^{(0)}=A B D, \quad y^{(0)}=y_{4} .
$$

Let us see what the outcome would be under the other procedures discussed in Appendix 1. Suppose that the supervisor appoints the strongest party, i.e., party $A$ to choose the government. Hence, if we apply the quick procedure, we get

$$
y^{(1)}=y_{3} .
$$

If we introduce negotiations, then party $A$ proposes government $y_{3}$, and hence, parties $B$ and $C$ have to react. Party $B$ says 'yes', since it prefers $y_{3}$ to $y_{4}$, and also party $C$ agrees, since $y_{3}$ is the only one government it belongs to. Hence, we also get

$$
y^{(2)}=y_{3} .
$$

Next, we apply the procedure of total gains.

$$
W_{A}^{\prime}=\frac{30}{85}, \quad W_{B}^{\prime}=\frac{20}{85}, \quad W_{C}^{\prime}=\frac{17}{85}, \quad W_{D}^{\prime}=\frac{18}{85},
$$

and therefore

$$
\sum_{i \in D M^{* *}} W_{i}^{\prime} \cdot B_{i}\left(y_{3}\right)=\frac{209}{425}, \quad \sum_{i \in D M^{* *}} W_{i}^{\prime} \cdot B_{i}\left(y_{4}\right)=\frac{216}{425} .
$$

Hence, the procedure based on total gain yields

$$
y^{(3)}=y_{4}
$$

Note that this method does not have to represent the preferences of the majority of parties from $D M^{* *}$, since party $D$ is the only one which prefers $y_{4}$ to $y_{3}$.

\section{References}

Austen-Smith, D., \& Banks, J. (1988). Elections, coalitions, and legislative outcomes. American Political Science Review, 82, 405-422. 
Axelrod, R. (1970). Conflict of interest: a theory of divergent goals with applications to politics. Chicago: Markham.

Bana e Costa, C. A., \& Vansnick, J. C. (1999). The MACBETH approach: Basic ideas, software and an application. In N. Meskens \& M. Roubens (Eds.), Advances in decision analysis (pp. 131-157). Dordrecht: Kluwer Academic.

Bana e Costa, C. A., De Corte, J. M., \& Vansnick, J. C. (2003). MACBETH (LSE OR Working Paper 03.56).

Baron, D. P. (1993). Government formation and endogenous parties. American Political Science Review, $87(1), 34-47$.

Carlsson, C., Ehrenberg, D., Eklund, P., Fedrizzi, M., Gustafsson, P., Merkuryeva, G., Riissanen, T., \& Ventre, A. (1992). Consensus in distributed soft environments. European Journal of Operational Research, 61, 165-185.

De Swaan, A. (1973). Coalition theories and cabinet formations. Amsterdam: Elsevier, North Holland.

De Vries, M. (1999). Governing with your closest neighbour: an assessment of spatial coalition formation theories. Print Partners Ipskamp.

Grofman, B. (1982). A dynamic model of protocoalition formation in ideological $n$-space. Behavioral Science, 27, 77-90.

Kahan, J., \& Rapoport, A. (1984). Theories of coalition formation. Lawrence Erlbauw Associates.

Kirchsteiger, G., \& Puppe, C. (1997). On the formation of political coalitions. Journal of Institutional and Theoretical Economics, 153, 293-319.

Laver, M., \& Schofield, N. (1990). Multiparty government: the politics of coalition in Europe. Oxford: Oxford University Press.

Laver, M., \& Shepsle, K. A. (1990). Coalitions and cabinet government. American Political Science Review, 3, 873-890.

Laver, M., \& Shepsle, K. A. (1996). Making and breaking governments; cabinet and legislatures in parliamentary democracies. Cambridge: Cambridge University Press.

McKelvey, R. D., Ordeshook, P. C., \& Winer, M. D. (1978). The competitive solution for n-person games without transferable utility: with an application for committee games. American Political Science Review, 72, 599-615.

Myerson, R. B. (1977). Graphs and cooperation in games. Mathematics of Operations Research, 2, $225-229$.

Peleg, B. (1981). Coalition formation in simple games with dominant players. International Journal of Game Theory, 10, 11-33.

Roubens, M., Rusinowska, A., \& De Swart, H. (2006). Using MACBETH to determine utilities of governments to parties in coalition formation. European Journal of Operational Research, 172(2), 588-603.

Rusinowska, A., De Swart, H., \& Van der Rijt, J. W. (2005). A new model of coalition formation. Social Choice and Welfare, 24, 129-154.

Schofield, N. (1993a). Political competition and multiparty coalition governments. European Journal of Political Research, 23, 1-33.

Schofield, N. (1993b). Party competition in a spatial model of coalition formation. In W. Barnett, M. Hinich \& N. Schofield (Eds.), Political economy: institutions, competition and representation. Cambridge: Cambridge University Press.

Schofield, N. (1995). Coalition politics: a formal model and empirical analysis. Journal of Theoretical Politics, 7(3), 245-281.

Shepsle, K. A. (1979). Institutional arrangements and equilibrium in multidimensional voting models. American Journal of Political Science, 23(1), 27-59.

Van Deemen, A. (1991). Coalition formation in centralized policy games. Journal of Theoretical Politics, 3 , 139-161.

Van Deemen, A. (1997). Coalition formation and social choice. Dordrecht: Kluwer Academic. 